\title{
Status of the MiniBooNE Experiment
}

\author{
Ion Stancu* ${ }^{\dagger}$ \\ Department of Physics and Astronomy \\ University of Alabama \\ Tuscaloosa, AL 35487-0324, USA \\ E-mail: 'ion.stancu@ua.edu'
}

Abstract: The MiniBooNE experiment at Fermilab has been designed to confirm or dismiss the LSND observation by looking for $\nu_{e}$ appearance in a $\nu_{\mu}$ beam. The experiment began taking beam data in September 2002. Here we describe the experiment, the first neutrino candidate events, and our expected sensitivity to a neutrino oscillation signal.

\section{Introduction}

Neutrino oscillations appear to be a widely-accepted phenomenon which successfully explains the solar (electron) neutrino deficit, as well as the atmospheric muon neutrino deficit. Moreover, the same deficits have been observed in artificial neutrino sources, as reported

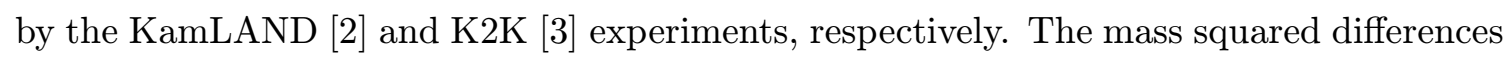
involved in these phenomena are $\Delta m_{\text {sol }}^{2} \approx 7 \times 10^{-5} \mathrm{eV}^{2} / \mathrm{c}^{4}$ and $\Delta m_{\text {atm }}^{2} \approx 3 \times 10^{-3} \mathrm{eV}^{2} / \mathrm{c}^{4}$, while the corresponding mixing angles appear to be nearly maximal. In addition, the LSND experiment, which ran at the Los Alamos National Laboratory from August 1993 until December 1998, has also reported evidence for neutrino oscillations in two channels: the decay-at-rest (DAR) channel $\bar{\nu}_{\mu} \rightarrow \bar{\nu}_{e}$, and the decay-in-flight (DIF) channel $\nu_{\mu} \rightarrow \nu_{e}$. The LSND final result [in] events after background subtraction, which corresponds to an oscillation probability of $(0.264 \pm 0.067 \pm 0.045) \%$. The allowed values in the $\left(\sin ^{2} 2 \theta, \Delta m^{2}\right)$ parameter space corresponding to this result are shown in Figure ${ }_{-i}^{1}$ Also shown are the $90 \%$ confidence level

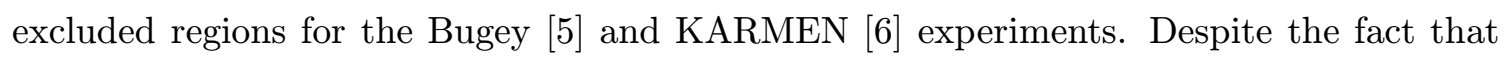
the KARMEN data appears to exclude a significant fraction of the LSND-favoured region, a combined analysis of the two data sets showed that practically this entire region is compatible with both experiments at the $90 \%$ confidence level [i]. Thus KARMEN's sensitivity did not allow it to make a definite statement regarding the LSND signal, neither confirming it, nor dismissing it.

\footnotetext{
${ }^{*}$ Speaker.

${ }^{\dagger}$ Representing the MiniBooNE collaboration [in|
} 


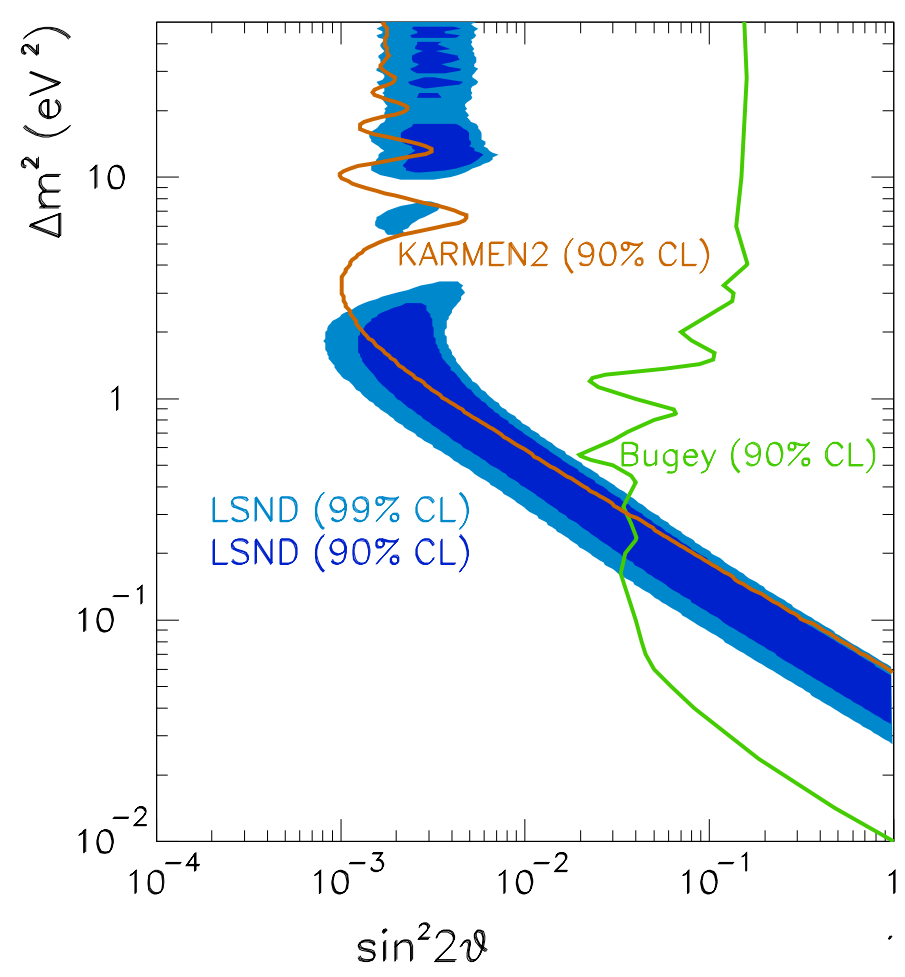

Figure 1: LSND allowed regions in the $\left(\sin ^{2} 2 \theta, \Delta m^{2}\right)$ parameter space. The light (dark) shaded regions correspond to the $99 \%(90 \%)$ confidence levels. Also shown are the $90 \%$ confidence level limits from the Bugey and KARMEN experiments.

The Booster Neutrino Experiment (BooNE) at Fermilab is a natural follow-up to the LSND experiment, and has been designed to confirm or dismiss the evidence for neutrino oscillations reported by the Los Alamos measurement. The first phase of the project, a single detector known as MiniBooNE (E-898), has become fully operational in September 2002. The experiment has two initial goals: (i) extend the search sensitivity for $\nu_{\mu} \rightarrow \nu_{e}$ oscillations by one order of magnitude in $\Delta m^{2}$ over previous searches; (ii) obtain several hundreds of events per year if the LSND signals are indeed due to neutrino oscillations. Moreover, should neutrino oscillations be observed, MiniBooNE itself can test for CP violation in the lepton sector by switching to an antineutrino $\left(\bar{\nu}_{\mu}\right)$ beam, while the full BooNE project would add a second detector, at a distance dictated by the data themselves, and carefully parameterize the $\nu_{\mu} \rightarrow \nu_{e}$ and $\bar{\nu}_{\mu} \rightarrow \bar{\nu}_{e}$ mixings.

\section{The Neutrino Beam}

The MiniBooNE neutrino beam is initiated by a primary beam of $8 \mathrm{GeV}$ protons from the Fermilab Booster accelerator incident on a 71-cm-long Be target within a magnetic horn focusing system, followed by a 50-m-long pion decay volume. The proton beam is delivered to the experiment at a rate of up to $5 \mathrm{~Hz}$ and an intensity of approximately $5 \times 10^{12}$ per spill, 
corresponding to an average current of about $3 \mu \mathrm{A}$. Each spill is made up of 84 buckets of beam every $18.8 \mathrm{~ns}$ for a total duration of $1.6 \mu \mathrm{s}$ - which allows for a very low cosmic-ray background in the detector. The Booster can reliably deliver protons for about two thirds of a calendar year, which allows the experiment to receive up to $5 \times 10^{20}$ protons on target (POT) per year.

The magnetic horn focuses secondary pions and kaons from the primary interactions. It operates at a current of $170 \mathrm{kA}$ for a pulse duration of $140 \mu \mathrm{s}$, producing a toroidal magnetic field that focuses $\pi^{+}$and defocuses $\pi^{-}$(or vice-versa). Therefore, a fairly pure $\nu_{\mu}$ or $\bar{\nu}_{\mu}$ beam can be produced, depending on the horn polarity. Figure $\underline{2}_{1}^{2}$ shows the shape of the expected neutrino fluxes at the location of the detector.

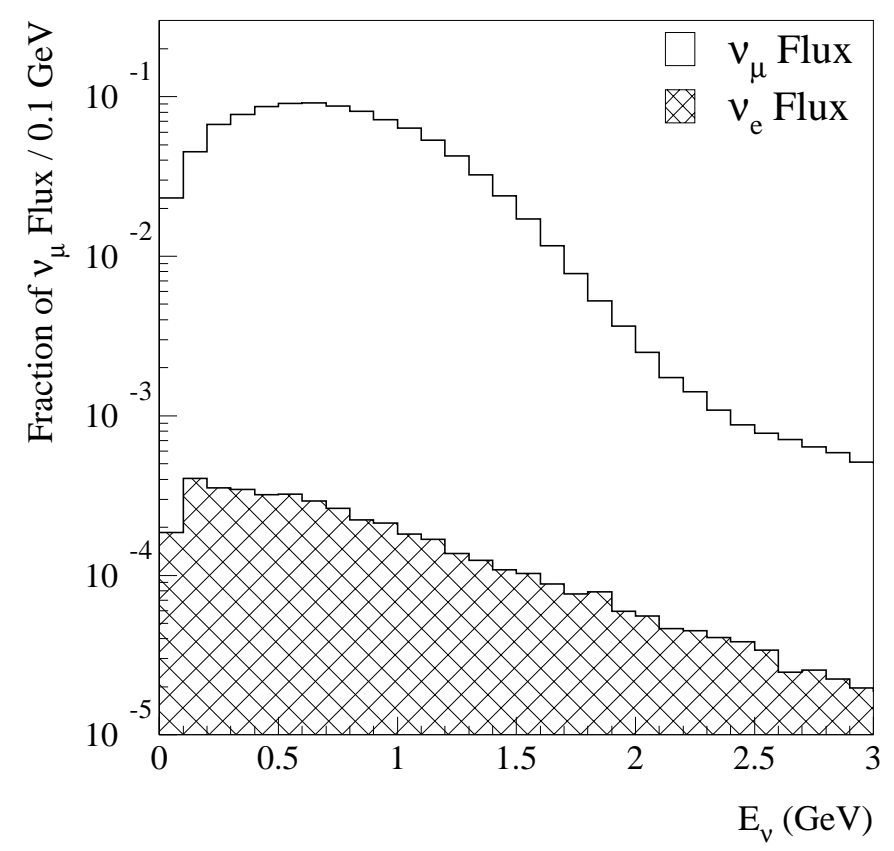

Figure 2: Calculated MiniBooNE neutrino fluxes at the location of the detector.

The neutrino flux at the detector will be determined using a variety of methods. Detailed simulations of the neutrino production processes have been performed and are ongoing. These simulations have been tuned using existing hadron production data, and will be complemented by data from the HARP experiment [8] MiniBooNE Be target and collected approximately 20 million triggers. A measurement of the $\nu_{\mu}$ charged-current rate in the detector will check the $\nu_{\mu}$ flux, as well as determine the energy distribution of the muons in the decay region - which contribute to the intrinsic $\nu_{e}$ background via $\mu^{+} \rightarrow e^{+} \nu_{e} \bar{\nu}_{\mu}$. The $\nu_{e}$ background from $K^{+} \rightarrow \pi^{0} e^{+} \nu_{e}$ decays will be determined by measuring the high-transverse-momentum muons from $K^{+} \rightarrow \mu^{+} \nu_{\mu}$ decays. An additional systematic check for a potential oscillation signal may be made by lowering an intermediate absorber half way into the 50-m-long decay region. This will change the signal to background ratio in a well-defined way, due to the different decay lengths of the 
pions and muons. For example, the $\nu_{e}$ background from $\mu^{+}$decay is concentrated at the downstream end of the decay pipe, while the $\nu_{e}$ background from $K^{+}$decay is concentrated at the upstream end.

\section{The Detector}

The MiniBooNE detector is located on the Fermilab site approximately 500 meters from the neutrino source. It consists of a spherical tank of radius $6.1 \mathrm{~m}$, lined with 12808 -inch photomultiplier tubes (PMTs) supported on an inner structure of $5.75 \mathrm{~m}$ radius, as shown in Figure $\overline{\beta_{i}}$. These PMTs point inward and provide approximately $10 \%$ photocathode

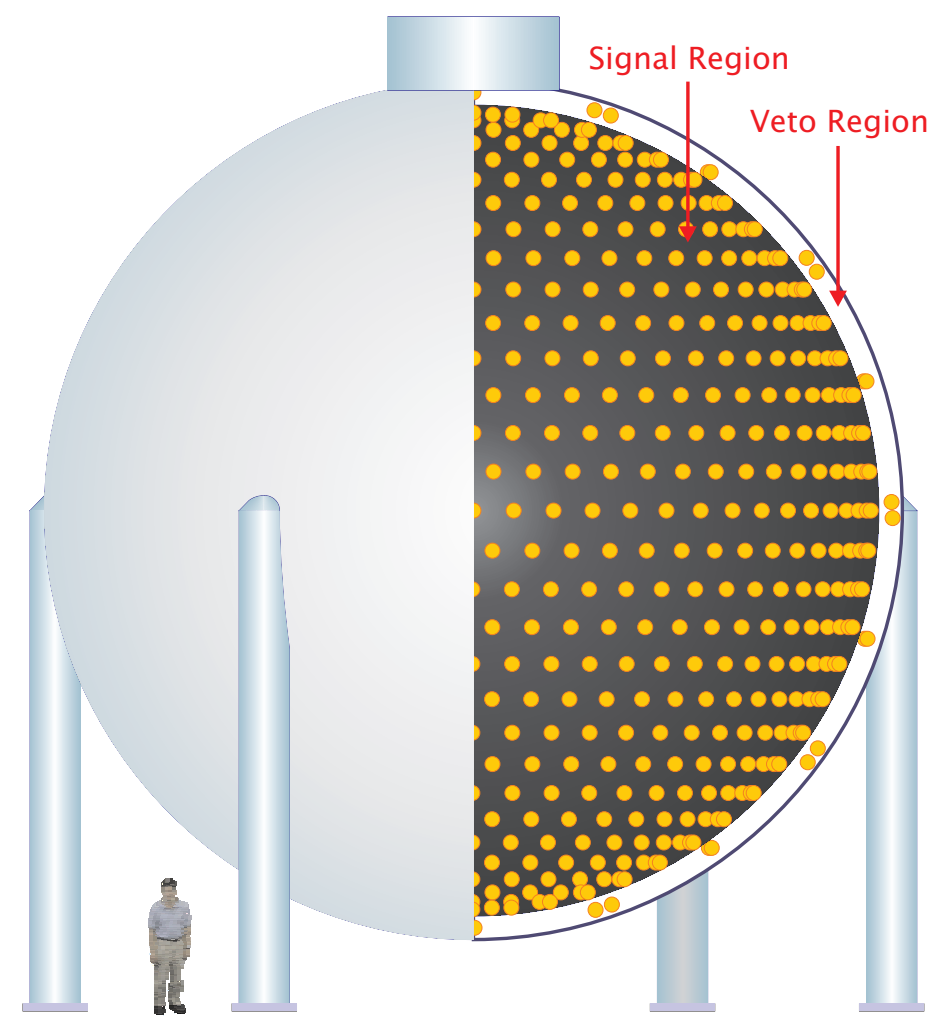

Figure 3: Schematic picture of the MiniBooNE detector showing the signal region and the optically isolated veto region.

coverage of the inner region of the detector. The PMT support structure also provides an optical barrier to create an outer veto region, which is viewed by 2408 -inch PMTs. The tank is filled with 800 tons of mineral oil, which provides an inner fiducial region of approximately 500 tons. The detector tank is housed in a cylindrical vault.

The MiniBooNE mineral oil (Exxon/Mobil Marcol 7) was selected primarily because of its high attenuation length, measured to be approximately 26 meters at $450 \mathrm{~nm}$. The calibration data collected so far indicate that the long attenuation length of the oil persists in the MiniBooNE tank. The oil has a density of $0.836 \mathrm{~g} / \mathrm{cm}^{3}$ and an index of refraction of 
1.46. This mineral oil produces some scintillation light, so both Čerenkov and scintillation light will be produced for particles with $\beta>0.68$. The total amount of light provides a good energy measurement for particles above and below the Čerenkov threshold. For $\beta=1$ particles about 4 photoelectrons are detected by the PMTs per MeV of energy loss. The scintillation light has an exponential time distribution with a time constant of approximately $18.5 \mathrm{~ns}$.

A circular room located above the tank vault houses the electronics, data acquisition (DAQ), oil circulation, and calibration systems. The entire structure is covered with a mound of earth to provide some cosmic-ray shielding. Each PMT is attached to one Teflon-jacketed cable which provides the high voltage (HV) and returns the signal as well. These cables are routed up and out of the tank through ports in the top cylindrical region of the tank to the DAQ system, where the signal is picked off the HV cable, amplified, and digitized. The DAQ hardware consists of custom-built cards in 13 VME crates which are read out via MVME2304 single-board computers (SBC) running LINUX. The data is zero suppressed by the SBC and shipped via ethernet to a single Intel-based computer running LINUX. This computer assembles the data and ships it to the Fermilab computer center where it is written to tape.

The trigger system consists of an additional VME crate housing custom-built cards that collect PMT multiplicity and beam information to form event triggers. The primary MiniBooNE trigger is simply a "beam-on-target" signal from the accelerator, which initiates a data readout in a $20 \mu \mathrm{s}$ window around the $1.6 \mu$ s beam spill (regardless of PMT multiplicity). Additional triggers are formed for calibration purposes and for a supernova search [i. using the detector multiplicity information. The DAQ hardware and software is built with data buffering capability at all levels to create a virtually dead-time-free system.

Calibration for the detector is obtained through three different calibration systems. A pulsed laser provides light to four different light-scattering flasks hanging in the inner region of the tank. This light is used to determine the time and gain calibrations of each of the inner PMTs. An array of seven scintillator cubes hanging in the main region of the tank are used to tag a sample of stopping muons in the tank which allows energy calibration with muon tracks of known length. A muon tracker system consisting of 2 horizontal planes of scintillator is installed above the tank. This tracker allows muon tracks of a well-known direction to be tagged and studied for direction calibration. In addition to these systems, the ubiquitous cosmic-ray muons provide a constant source of data with which to study and calibrate the detector. In particular, the stopped cosmic-ray muons provide, through their subsequent decays, an invaluable source of electrons as cross-checks of the Monte Carlo (MC) simulations, energy scale measure, and energy resolution. Preliminary studies indicate an electron energy resolution of about $14 \%$ at the Michel electron endpoint energy $(52.8 \mathrm{MeV})$.

\section{The MiniBooNE Neutrino Oscillations Search}

The $\nu_{\mu} \rightarrow \nu_{e}$ oscillation search will be conducted by measuring the event rate for the $\nu_{e}$ induced reaction, $\nu_{e} C \rightarrow e^{-} X$, and comparing to the rate expected from background 
processes. If the LSND result is indeed due to neutrino oscillations, approximately 1000 $\nu_{e}$-induced events are expected due to $\nu_{\mu} \rightarrow \nu_{e}$ oscillations in two calendar years of running $\left(10^{21}\right.$ protons on target). The three main backgrounds to this search are: the intrinsic $\nu_{e}$ background in the beam from $\mu$ and $K$ decay in the decay pipe, mis-identification of $\mu$ events $\left(\nu_{\mu} C \rightarrow \mu^{-} X\right)$, and mis-identification of $\pi^{0}$ events $\left(\nu_{\mu} C \rightarrow \nu_{\mu} \pi^{0} X\right)$. The number of events for signal and backgrounds are listed in Table $\underset{1}{1}$. These backgrounds will be measured.

\begin{tabular}{|c|c|c|}
\hline Process & Reaction & Number of events \\
\hline \hline LSND-based $\nu_{\mu} \rightarrow \nu_{e}$ signal & $\nu_{e} C \rightarrow e^{-} X$ & 1000 \\
Intrinsic $\nu_{e}$ background & $\nu_{e} C \rightarrow e^{-} X$ & 1500 \\
Mis-ID $\mu^{-}$background & $\nu_{\mu} C \rightarrow \mu^{-} X$ & 500 \\
Mis-ID $\pi^{0}$ background & $\nu_{\mu} C \rightarrow \nu_{\mu} \pi^{0} X$ & 500 \\
\hline$\nu_{\mu} C$ charged-current scattering & $\nu_{\mu} C \rightarrow \mu^{-} X$ & 500,000 \\
$\nu_{\mu} C$ neutral-current $\pi^{0}$ production & $\nu_{\mu} C \rightarrow \nu_{\mu} \pi^{0} X$ & 50,000 \\
$\nu_{\mu} e$ elastic scattering & $\nu_{\mu} e \rightarrow \nu_{\mu} e$ & 100 \\
\hline \hline
\end{tabular}

Table 1: Estimated number of neutrino oscillation signal and background events after 2 years of data taking with neutrinos $\left(10^{21}\right.$ protons on target). Also shown are the number of events from other neutrino reactions in MiniBooNE.

As described in Section 2, the number of events from intrinsic $\nu_{e}$ that are produced from $\mu^{+}$decays in the target region will be determined from $\nu_{\mu}$ charged-current scattering in

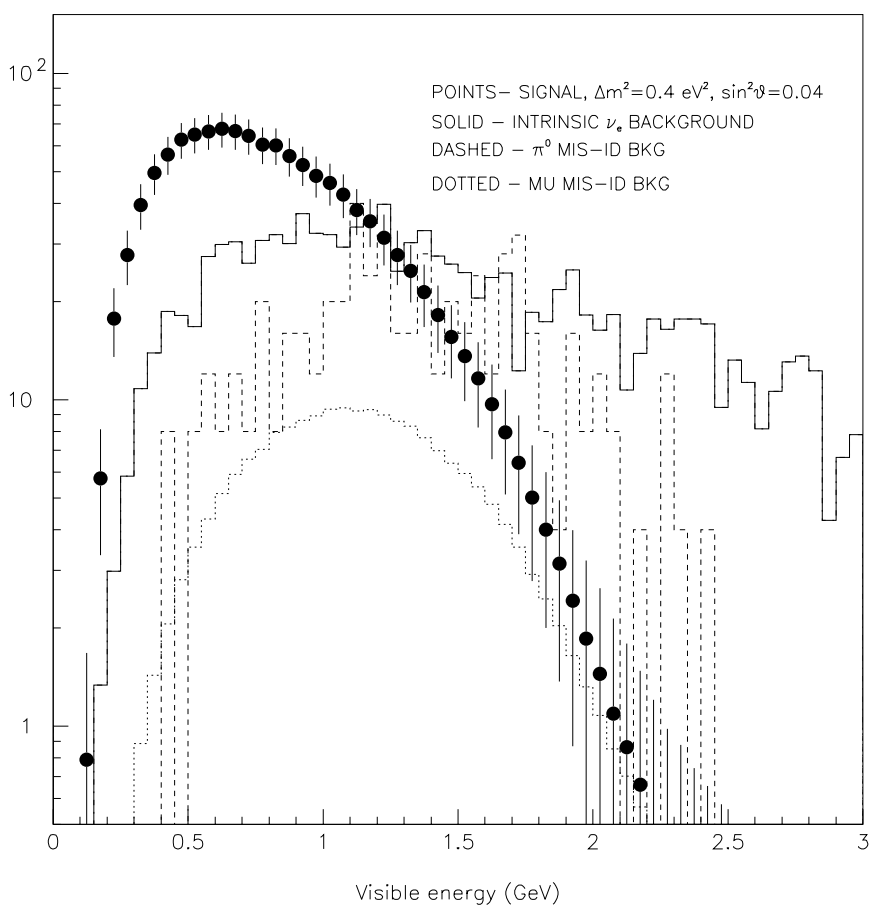

Figure 4: MiniBooNE expected signal and background energy distributions for the case of $\Delta m^{2}=$ $0.4 \mathrm{eV}^{2} / \mathrm{c}^{4}$ and $\sin ^{2} 2 \theta=0.04$. 
the detector. Also, the number of intrinsic $\nu_{e}$ from $K^{+}$decays will be determined from the $\mu$ detected with the off-axis LMC spectrometer. The number of $\mu^{-}$and $\pi^{0}$ events mis-identified as $e^{-}$events will be measured via the large number of correctly identified $\nu_{\mu} C \rightarrow \mu^{-} X$ and $\nu_{\mu} C \rightarrow \nu_{\mu} \pi^{0} X$ events, respectively. Figure $\overline{4}_{1}^{\overline{4}}$ shows the expected signal and background energy distributions for the case of $\Delta m^{2}=0.4 \mathrm{eV}^{2} / \mathrm{c}^{4}$ and $\sin ^{2} 2 \theta=0.04$, while Fig. ${ }_{1}^{5}$ illustrates the expected sensitivity to neutrino oscillations for $10^{21}$ protons on target with the horn either in the $\nu_{\mu}$ or in the $\bar{\nu}_{\mu}$ configuration.

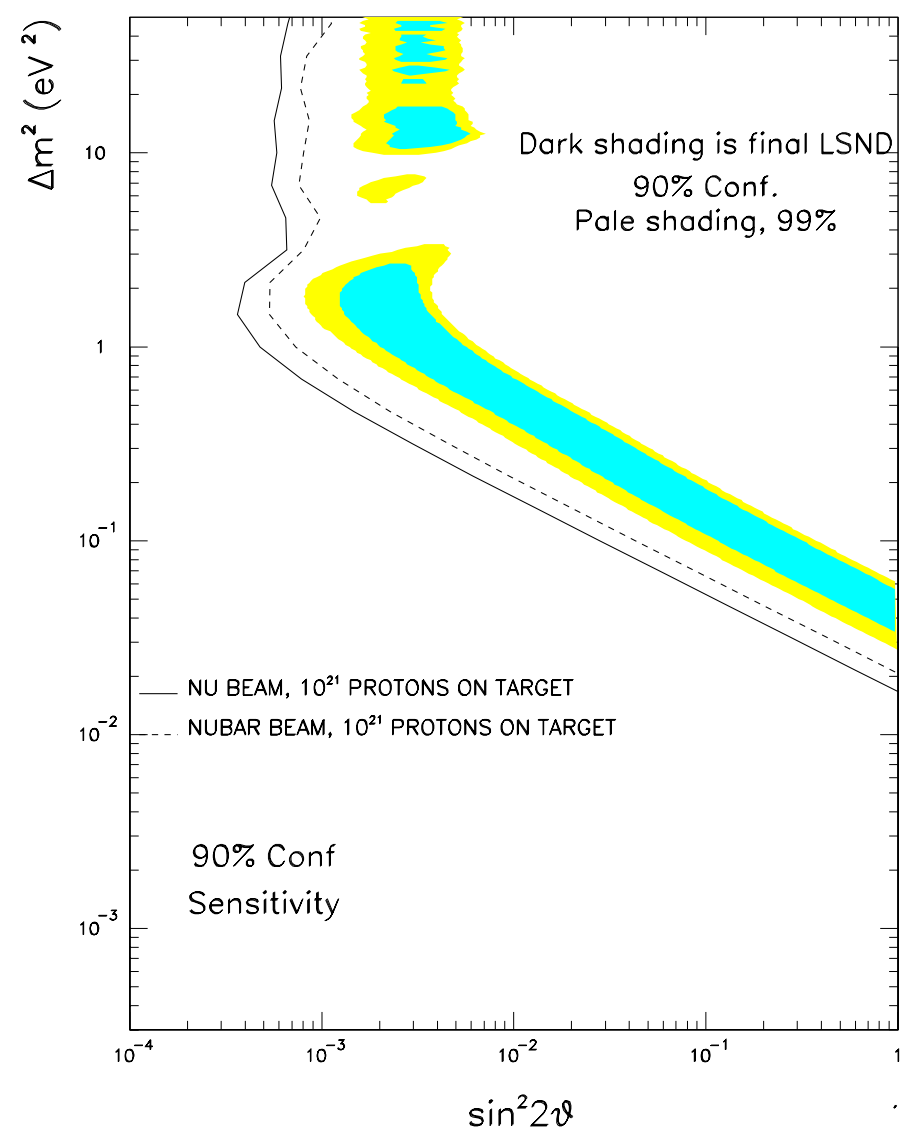

Figure 5: MiniBooNE expected oscillation sensitivity for 2 years of $\nu_{\mu}$ or $\bar{\nu}_{\mu}$ running.

\section{Preliminary Data}

The MiniBooNE detector and beam have been fully operational for almost a full year now, and data taking has been going very smoothly. The detector has been calibrated with laser calibration events, the energy scale and resolution have been determined from cosmic-ray muons and Michel electron events, and approximately 160, 000 clean neutrino events have been recorded with $1.5 \times 10^{20}$ protons on target. Figure $\overline{6}_{1}$ below illustrates the simple selection criteria which reduce the beam off backgrounds to a $10^{-3}$ level: a veto shield multiplicity cut $N_{\text {veto }}<6$ eliminates the cosmic-ray muons, while a tank multiplicity cut 

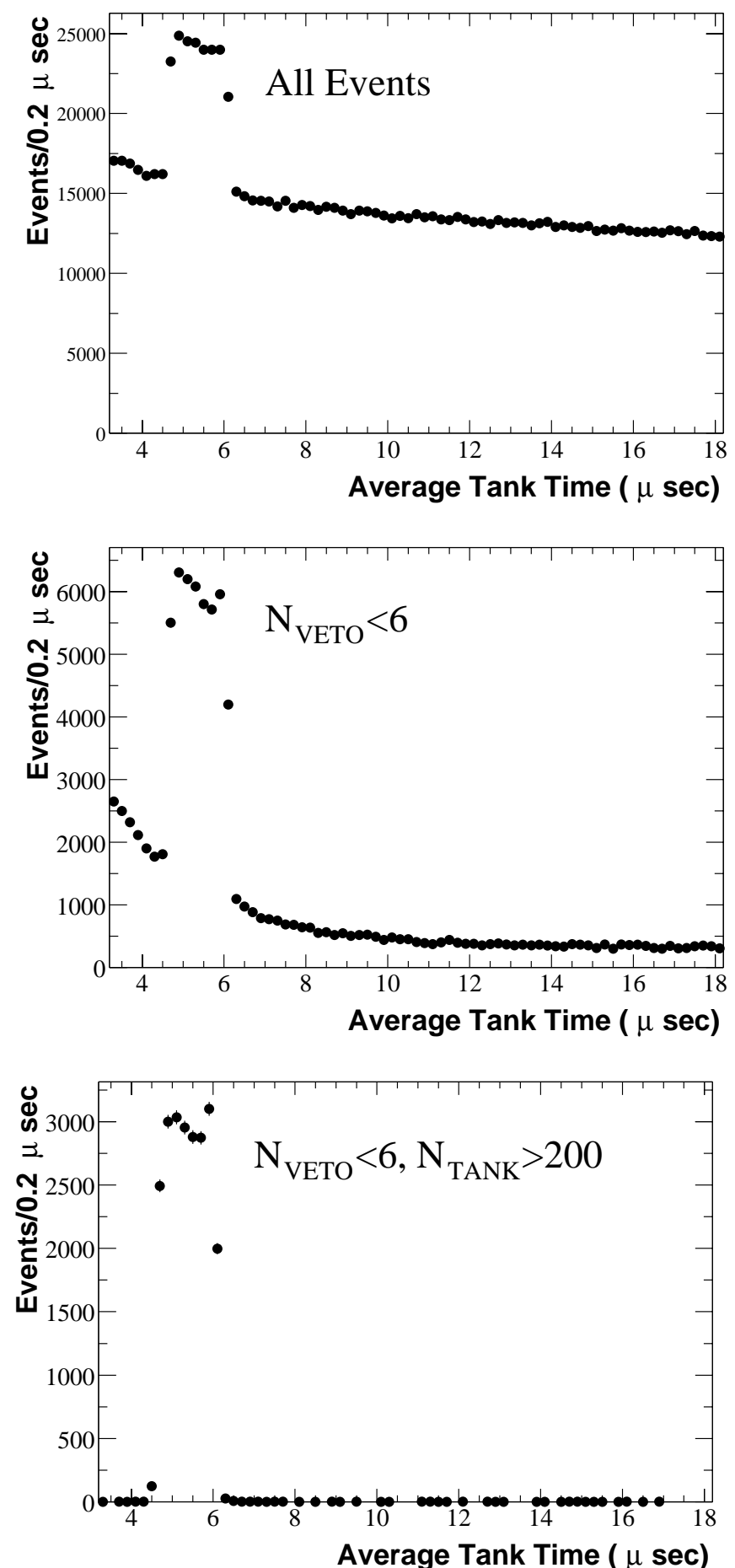

Figure 6: MiniBooNE event distribution in and around the beam window before and after the simple selection criteria which reduce the beam off background.

$N_{\text {tank }}>200$ eliminates the Michel electrons from the decay of the stopped cosmic-ray muons. The beam pulse width of about $1.6 \mu \mathrm{s}$ is in clearly seen in the figure. 
The $\nu_{e}$ appearance search will be a blind analysis. Consequently, despite the fact that MiniBooNE can clearly identify the beam-induced events, one is allowed to either look at all information for some events or some information for all events, but not all information for all events. Meanwhile, in parallel to continuing understanding the detector response and tuning the $\mathrm{MC}$ simulations, the experiment is concentrating on other physics analyses which are not only interesting in their own right, but they are also necessary for the $\nu_{\mu} \rightarrow \nu_{e}$ oscillations analysis: they will check the data/MC agreement, the reliability of the reconstruction and particle-identification algorithms, and provide understanding of the beam-induced backgrounds.

MiniBooNE is clearly identifying charged-current quasi-elastic $\nu_{\mu} C \rightarrow \mu X$ events. These events have a relatively high abundance (about 40\%), a simple topology, and can be identified with a relatively high efficiency and purity (of approximately $30 \%$ and $90 \%$, respectively). The muons are forward peaked along the incident neutrino beam direction,
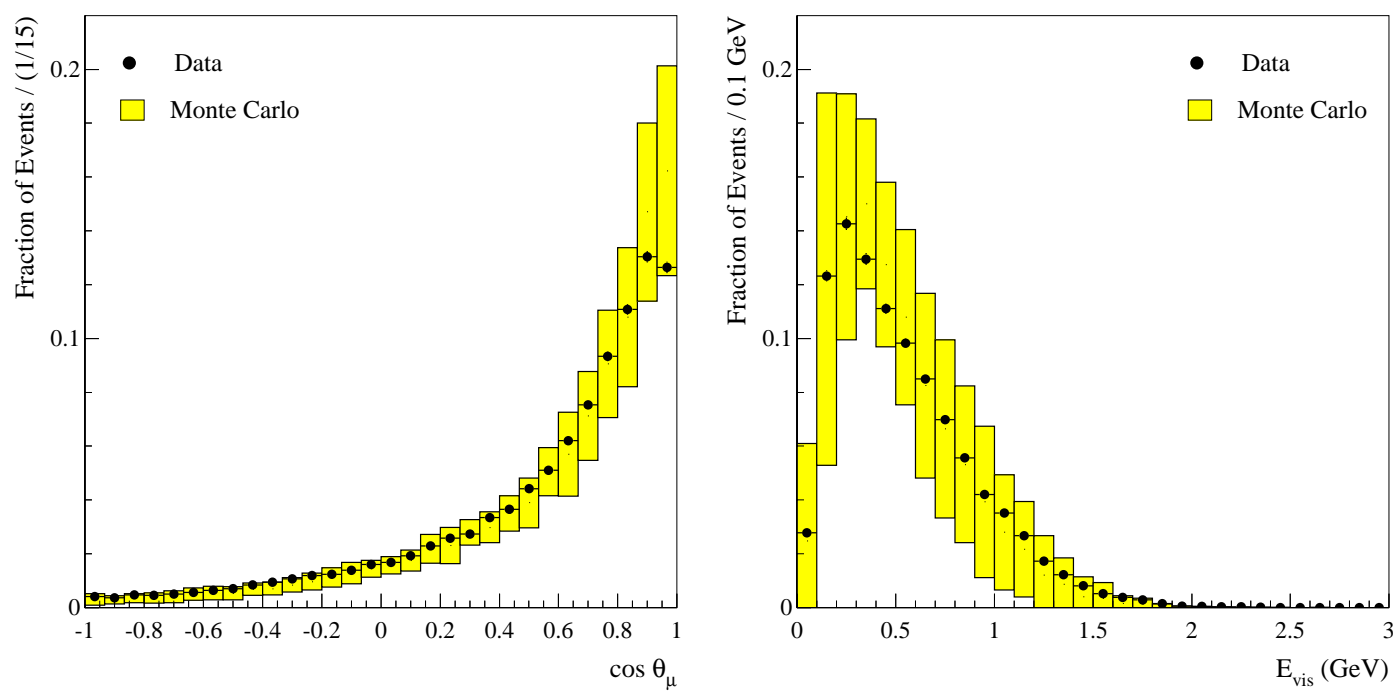

Figure 7: MiniBooNE reconstructed event direction with respect to the incident neutrino beam and visible (electron-equivalent) energy distributions for $\nu_{\mu} C$ charged-current quasi-elastic events.

as clearly seen in Fig. $\bar{T}_{1}$ above. The predicted MC distribution is shown superimposed in the same figure, with our current (conservative) estimates for the error bars; they include errors in the neutrino fluxes, cross sections and optical parameters of the detector medium. Both distributions have been normalized to unit area. The visible energy distribution is also in good agreement with the MC expectations, as illustrated in the same figure.

From a simple kinematic reconstruction one can use the reconstructed muon energy and direction to calculate the incident neutrino energy and also the momentum transfer. These quantities are shown in Fig. $\left.\right|_{-1} ^{\overline{8}}$ along with the MC predictions with a relative normalization. The lower-than-predicted data values at low $Q^{2}$ are currently under investigation and can be influenced by a variety of factors, such as nuclear effects, nuclear form factors, etc. This effect is also related to the lower-than-predicted number of events in the most forward direction (first bin in the $\cos \theta_{\mu}$ distribution of Fig. $\left.\overline{\underline{T}}_{1}\right)$. 

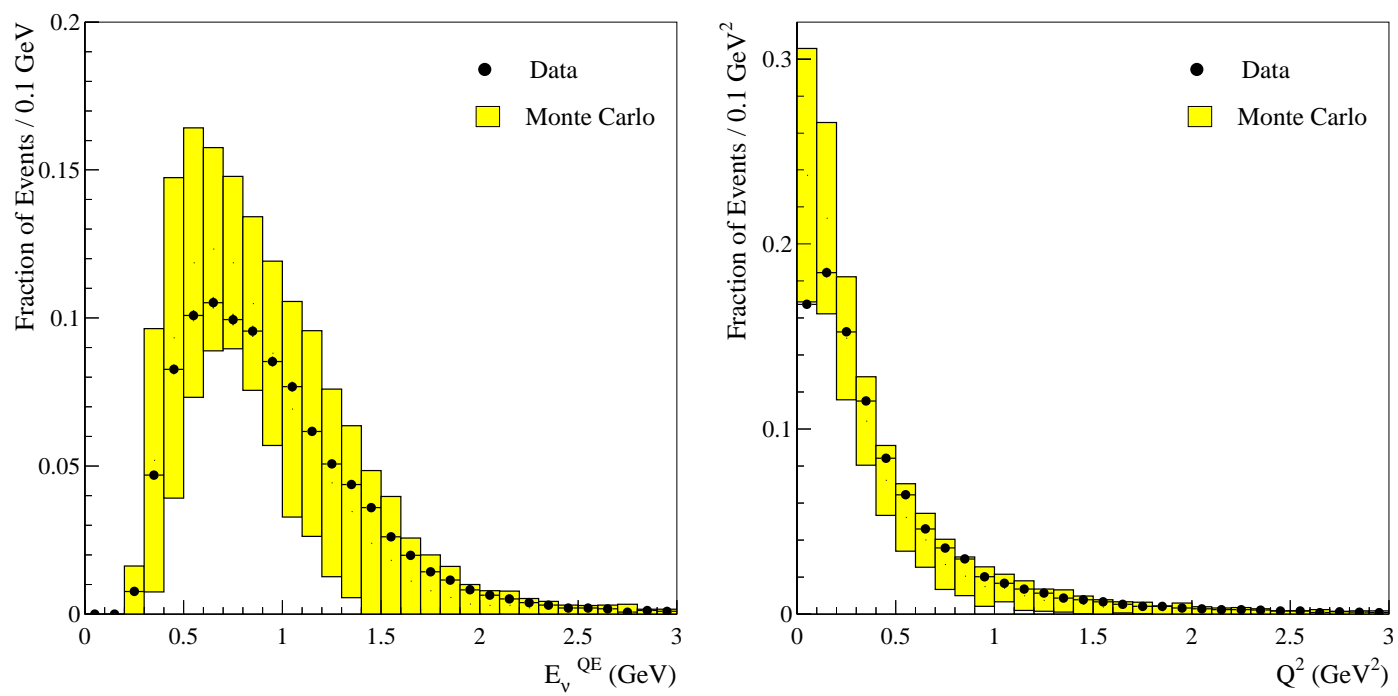

Figure 8: MiniBooNE reconstructed incident neutrino energy and $Q^{2}$ distributions for $\nu_{\mu} C$ charged-current quasi-elastic events.

The reconstructed incident neutrino energy resolution is shown in Fig. 'i ${ }_{-1}^{-1}$ below as obtained from our current MC simulations. The plot indicates a resolution of better than $10 \%$ for neutrino energies above $800 \mathrm{MeV}$.

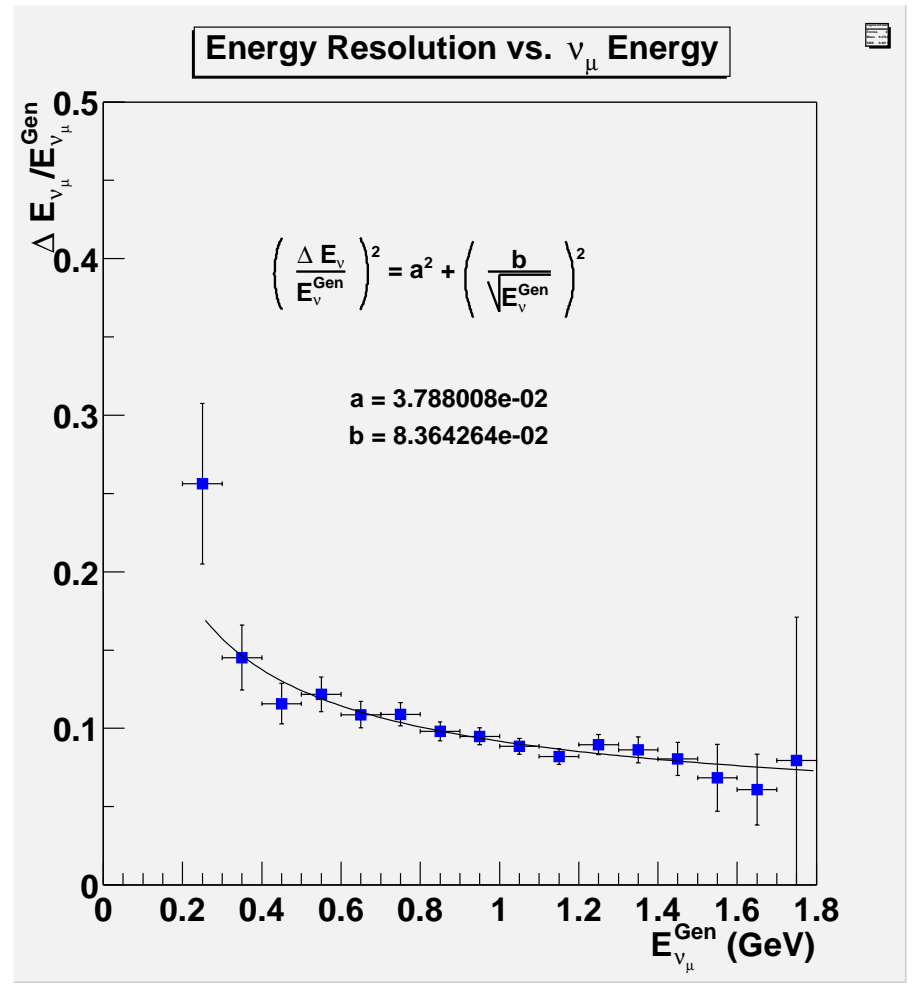

Figure 9: MiniBooNE reconstructed neutrino energy resolution. 
MiniBooNE is also clearly identifying and reconstructing neutral current $\pi^{0}$ events from either coherent production $\nu_{\mu} C \rightarrow \nu_{\mu} \pi^{0} X$, or resonant production $\nu_{\mu}(p / n) \rightarrow \nu_{\mu} \Delta$ and the subsequent $\Delta$ decay. These events have a characteristic two ring topology (from the $\pi^{0} \rightarrow \gamma \gamma$ decay), and the invariant $\pi^{0}$ is reconstructed from the reconstructed energies of the two photons, $E_{1}$ and $E_{2}$, and their relative angle, $\theta_{12}$ :

$$
m_{\pi}^{2}=2 E_{1} E_{2}\left(1-\cos \theta_{12}\right) .
$$

The distribution of the reconstructed invariant mass is shown in Fig. 'i $1 \overline{0} \bar{i}$ below, which yields a mass resolution of about $21 \mathrm{MeV}$. The events contributing to this sample have

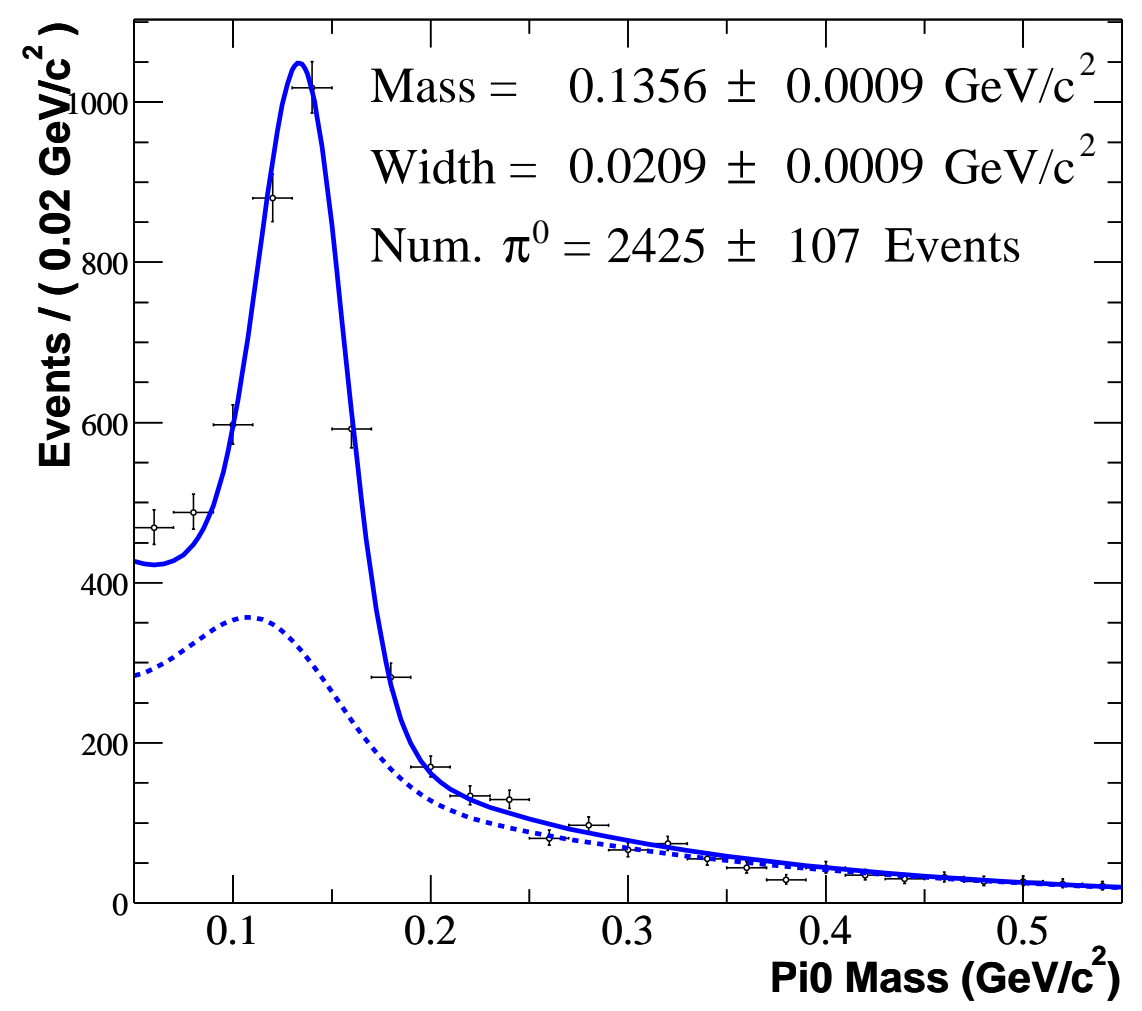

Figure 10: MiniBooNE reconstructed $\pi^{0}$ mass distribution. The dashed curve denotes the expected background from MC simulations, which is also peaked.

the standard $N_{\text {veto }}<6$ and $N_{\text {tank }}>200$ selection criteria applied, the event vertex must reconstruct at least $50 \mathrm{~cm}$ away from the surface of the PMTs, and each ring must have at least an electron-equivalent energy of $40 \mathrm{MeV}$.

Finally, a third event sample of special interest to us is the neutral current elastic scattering

$$
\nu_{\mu}+(p / n) \rightarrow \nu_{\mu}+(p / n) .
$$

These events have an abundance of approximately $15 \%$ and completely dominate the low tank hit multiplicity regime of the expected data set, as shown in Fig. 


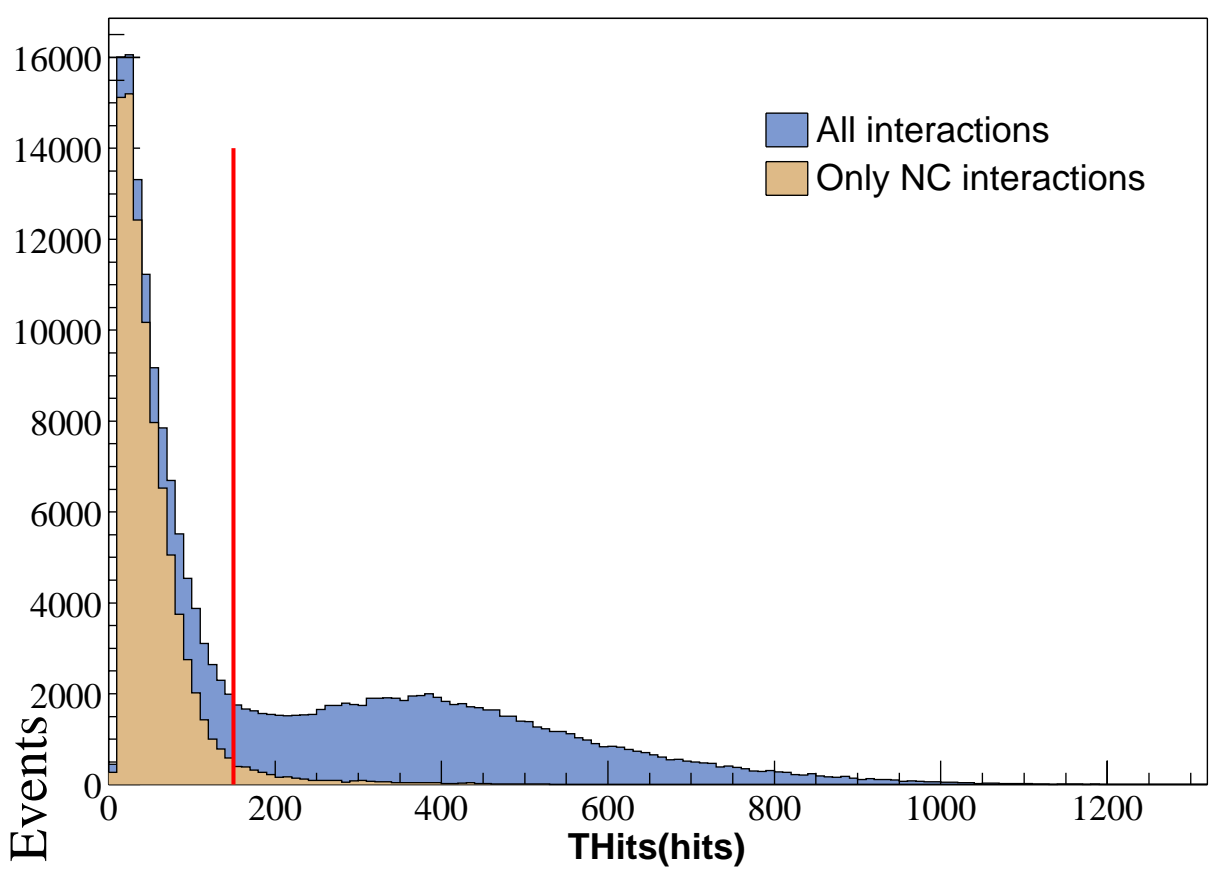

Figure 11: MiniBooNE tank hit multiplicity distribution for neutral current elastic scattering events only as compared to that for all events (MC data).

tion, these events are mostly sub-Čerenkov, and thus they are dominated by scintillation light. Consequently, this data set serves as a primary calibration tool for understanding the optical properties of scintillation light in the detector medium (attenuation length, rescattering fraction, time constant, etc.). In addition, this event sample (in particular the proton-to-neutron ratio) is sensitive to the strange quark contribution to the nucleon spin. A comparison of the tank hit multiplicity distribution between data and MC is shown in Fig. ${ }_{1}^{1} \overline{2}$, normalized above 50 tank hits. The events have been selected by requiring a relatively high fraction of scintillation light. The disagreement below 50 tank hits is currently under investigation.

\section{Conclusions}

The MiniBooNE detector and beam line have been operating smoothly for a year now and we are in the process of analyzing different physics channels, while continuously improving our understanding of the detector response and MC simulations. Despite the fact that the total number of protons on target after the first year of operation is a factor of three below the design intensity, we are convinced that the modifications and improvements to the Fermilab Booster will bring the neutrino beam to the required level after the Oct/Nov2003 shutdown. 


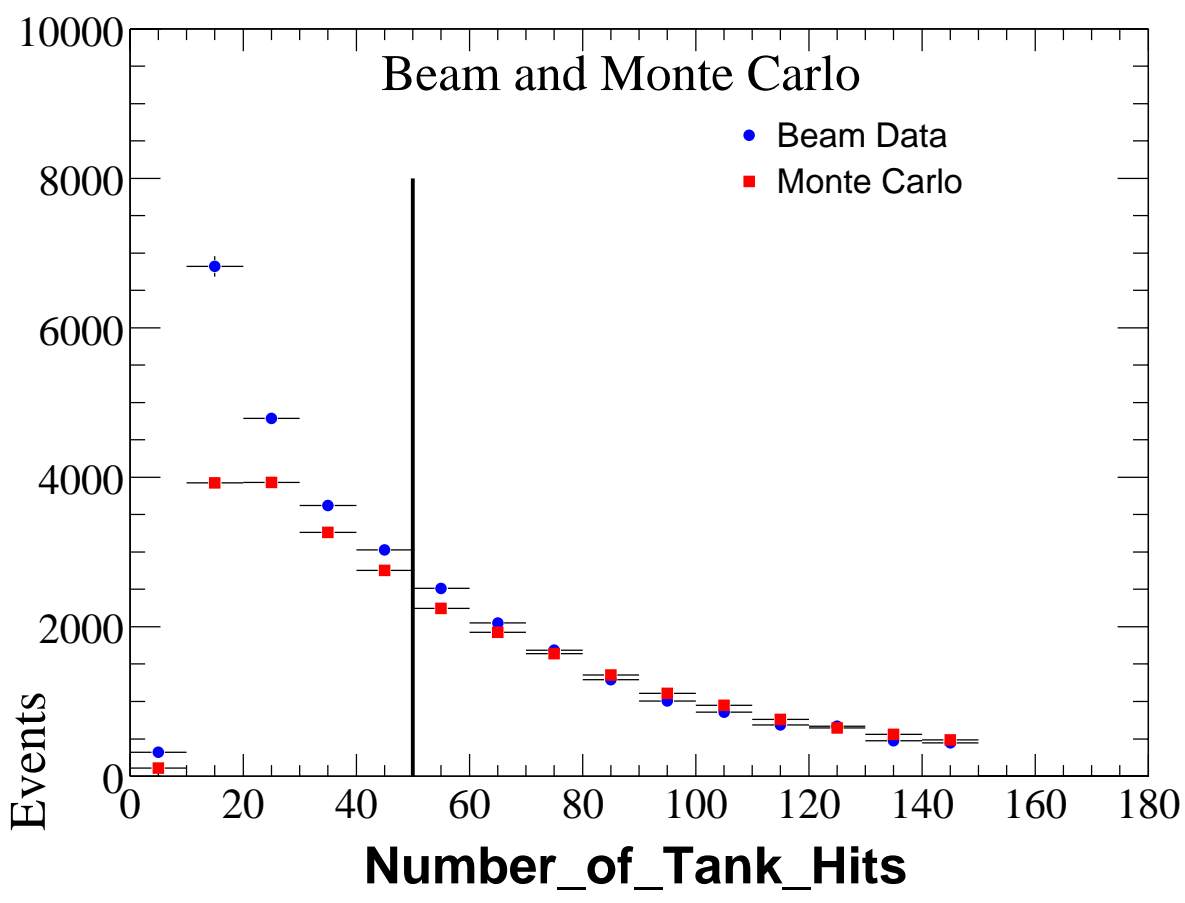

Figure 12: MiniBooNE tank hit multiplicity distribution for neutral current elastic scattering events (data and MC). The distributions have been normalized above 50 tank hits.

The current plan is to run in the $\nu_{\mu}$ mode until MiniBooNE collects $5 \times 10^{20}$ protons on target, with the possibility of changing to the $\bar{\nu}_{\mu}$ mode afterwards and also $25 \mathrm{~m}$ absorber running. The future MiniBooNE schedule is dependent on the number of protons delivered per year to the experiment. First oscillations results are expected by 2005, and if the LSND signal is confirmed, an initial determination of the oscillation parameters can be made. A second detector (BooNE) will then be built at a different distance in order to obtain the highest precision measurement of the oscillation parameters. The neutrino flux goes as $r^{-2}$ to very good approximation, so that a simple ratio of events in the two detectors as a function of energy will cancel most of the systematic uncertainties and will allow $\Delta m^{2}$ to be measured to about $\pm 0.02 \mathrm{eV}^{2} / \mathrm{c}^{4}$.

\section{Acknowledgments}

This work has been partially supported by US/DoE grant \# DE-FG02-03ER41261.

\section{References}

[1] The BooNE collaboration consists of the following institutions: University of Alabama, Bucknell University, University of Cincinnati, University of Colorado, Columbia University, Embry Riddle Aeronautical University, Fermi National Accelerator Laboratory, Indiana 
University, Los Alamos National Laboratory, Louisiana State University, University of Michigan and Princeton University.

[2] K. Eguchi et al. (KamLAND Collaboration), 'Phys. Rev. Lett. 90 (2003) 021802,

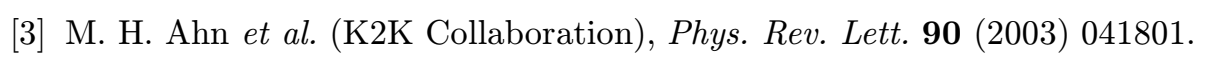

[4] A. Aguilar et al. (LSND Collaboration), Phys. Rev. D' $\mathbf{6} \overline{4}(\overline{2} 001) 1 \overline{1} \overline{2} 0 \overline{7}$.

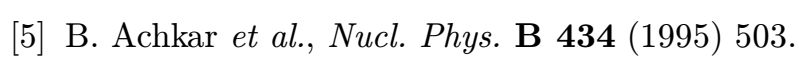

[6] B Armbruster et al. (KARMEN Collaboration), 'Phys. Rev. D' 65.

[7] E. D. Church et al., Phys. Rev. D'66 $(2002) 013001$ i

[8] "Proposal to study hadron production for the neutrino factory and for the atmospheric neutrino flux", M. G. Catanesi et al., CERN-SPSC/99-35 (1999).

[9] J. Beacom, proceedings of "Neutrino 2002"; M. K. Sharp et al., 'Phys. Rev. D. $6 \mathbf{6} 6(2002) !$ :- 